\title{
Zur Kurvendiskussion der Potenzsummenpolynome
}

Dietmar Treiber

Herrn G. Pickert in Dankbarkeit gewidmet

Dietmar Treiber studierte in Köln Mathematik und Physik und promovierte dort 1971 über $p$-adische Analysis. Er unterrichtet Mathematik und Informatik an einem Gymnasium. Intensiv befasst er sich mit mathematikdidaktischen Fragen in Analysis und Wahrscheinlichkeitsrechnung und mit dem Einsatz von Computern im Mathematikunterricht. Besonders gern beschäftigt er sich mit Elementarmathematik.

\section{Einleitung}

Für alle $n \in \mathbb{N}$ bezeichnen wir mit $P_{n}$ das $n$-te Potenzsummenpolynom, das dadurch gekennzeichnet ist, daß für alle ganzahligen $x \geq 2$ die Gleichung

$$
P_{n}(x)=1^{n-1}+2^{n-1}+3^{n-1}+\cdots+(x-1)^{n-1}
$$

erfüllt ist. Es gilt dann:

$$
\begin{aligned}
& P_{1}(x)=x \\
& P_{2}(x)=\frac{1}{2} x^{2}-\frac{1}{2} x \\
& P_{3}(x)=\frac{1}{3} x^{3}-\frac{1}{2} x^{2}+\frac{1}{6} x
\end{aligned}
$$

Die Formeln, welche die Summen der ersten, zweiten, dritten etc. Potenzen der ganzen Zahlen darstellen, sind beliebte Themen im Mathematikunterricht des Gymnasiums. Schon Jakob Bernoulli und wenig später Leonard Euler haben die zugehörigen, durch diese Formeln definierten reellen Polynome untersucht. Das Interesse ergab sich aus der Tatsache, dass die Koeffizienten dieser Polynome in enger Beziehung zu den sogenannten Bernoullizahlen stehen, die an verschiedensten Stellen der Mathematik in geradezu wundersamer Weise auftreten, so etwa bei den Potenzreihe des Tangens und des Cotangens oder bei der Berechnung von Werten der Zetafunktion. - Dietmar Treiber zeigt in seinem Beitrag, wie mit ganz einfachen und an der Schule bekannten Hilfsmitteln der Differential- und Integralrechnung interessante Eigenschaften dieser Polynome hergeleitet werden können. ust 


$$
\begin{aligned}
& P_{4}(x)=\frac{1}{4} x^{4}-\frac{1}{2} x^{3}+\frac{1}{4} x^{2} \\
& P_{5}(x)=\frac{1}{5} x^{5}-\frac{1}{2} x^{4}+\frac{1}{3} x^{3}-\frac{1}{30} x \\
& P_{6}(x)=\frac{1}{6} x^{6}-\frac{1}{2} x^{5}+\frac{5}{12} x^{4}-\frac{1}{12} x^{2} \\
& P_{7}(x)=\frac{1}{7} x^{7}-\frac{1}{2} x^{6}+\frac{1}{2} x^{5}-\frac{1}{6} x^{3}+\frac{1}{42} x .
\end{aligned}
$$

Kern dieses Aufsatzes ist eine neue Rekursionsformel für die Potenzsummenpolynome. Die Formel verwendet Differential- und Integralrechnung und ermöglicht es, mit ganz elementarer Analysis Neues über diese Polynome herauszufinden und Bekanntes einfach herzuleiten. Unser wichtigstes Ergebnis besagt, daß alle Potenzsummenpolynome punktoder achsensymmetrisch sind.

Die Potenzsummenpolynome sind eng verbunden mit den Bernoullischen Zahlen. Als Nebenergebnis gewinnen wir eine anschauliche Deutung dieser Zahlen und weisen wichtige Eigenschaften dieser Zahlen elementar nach.

\section{Potenzsummenpolynome und Bernoullische Zahlen}

Eine einheitliche Darstellung der Potenzsummenpolynome fand Jakob Bernoulli (siehe [1]). Die nach ihm benannten Bernoullischen Zahlen $B_{0}, B_{1}, B_{2}, \ldots$ sind rekursiv definiert durch $B_{0}=1$ und

$$
\sum_{i=0}^{n-1}\left(\begin{array}{c}
n \\
i
\end{array}\right) B_{i}=0
$$

für alle $n \in \mathbb{N}$ mit $n \geq 2$. Z. B. gilt:

$$
B_{0}=1, B_{1}=-\frac{1}{2}, B_{2}=\frac{1}{6}, B_{3}=0, B_{4}=-\frac{1}{30}, B_{5}=0, B_{6}=\frac{1}{42} .
$$

Mit diesen Zahlen lassen sich die Potenzsummenpolynome berechnen durch

$$
P_{n}(x)=\frac{1}{n} \sum_{i=0}^{n-1}\left(\begin{array}{c}
n \\
i
\end{array}\right) B_{i} x^{n-i}
$$

für alle $n \in \mathbb{N}$.

Umgekehrt lassen sich die Bernoullischen Zahlen aus den Potenzsummenpolynomen als geometrische Größen zurückgewinnen, und zwar auf ganz verschiedene Arten. Sie sind z. B. Tangentensteigungen dieser Polynome. Denn es gilt $P_{n}^{\prime}(0)=B_{n-1}$ für alle $n \in \mathbb{N}$. Für uns ist hier eine andere geometrische Deutung der Bernoullischen Zahlen wesentlich:

Satz 1 Für alle $n \in \mathbb{N}$ ist $-\frac{B_{n}}{n}$ gleich dem Mittelwert von $P_{n}$ auf $[0,1]$.

Beweis. Für $n=1$ ergibt sich die Behauptung durch einfache Rechnung. Für alle $n \in \mathbb{N}$ mit $n \geq 2$ gilt:

$$
n \int_{0}^{1} P_{n}(x) d x=\sum_{i=0}^{n-1}\left(\begin{array}{c}
n \\
i
\end{array}\right) B_{i} \frac{1}{n+1-i}=\frac{1}{n+1} \sum_{i=0}^{n-1}\left(\begin{array}{c}
n+1 \\
i
\end{array}\right) B_{i}=-B_{n} .
$$




\section{Eine Rekursionsformel für die Potenzsummenpolynome}

$\mathrm{Da}$ man die Potenzsummenpolynome auch rekursiv berechnen kann, ist bekannt. Eine Rekursionsformel findet man z. B. in [5; S. 501]. Wir bemerken, daß diese Formel nur arithmetische Rechnungen verwendet. Daneben gibt es - wie wir nun zeigen noch eine ganz andere rekursive Beziehung zwischen den Potenzsummenpolynomen. Diese verwendet Differential- und Integralrechnung und eröffnet neue Möglichkeiten zur Untersuchung dieser Polynome mit elementarer Analysis.

Versuchen wir, durch Differenzieren eine Rekursionsformel für die Potenzsummenpolynome zu finden. Für alle $n \in \mathbb{N}$ gilt:

$$
\begin{aligned}
P_{n+1}^{\prime}(x) & =\frac{1}{n+1} \sum_{i=0}^{n}\left(\begin{array}{c}
n+1 \\
i
\end{array}\right) B_{i}(n+1-i) x^{n-i} \\
& =\sum_{i=0}^{n}\left(\begin{array}{c}
n \\
i
\end{array}\right) B_{i} x^{n-i} \\
& =n P_{n}(x)+B_{n} .
\end{aligned}
$$

Hieraus und aus Satz 1 folgt:

$$
P_{n+1}^{\prime}(x)=n\left(P_{n}(x)-\int_{0}^{1} P_{n}(t) d t\right) .
$$

Die Potenzsummenpolynome haben sämtlich an der Stelle 0 den Wert 0, wie Bernoullis Formel zeigt. Daher folgt aus der letzten Gleichung durch Integrieren:

Rekursionsformel Für alle $n \in \mathbb{N}$ gilt:

$$
P_{n+1}(x)=n\left(\int_{0}^{x} P_{n}(t) d t-x \int_{0}^{1} P_{n}(t) d t\right) .
$$

\section{Die Symmetrie der Potenzsummenpolynome}

Unsere Rekursionsformel ist ein Schlüssel zum Verständnis des Symmetrieverhaltens der Potenzsummenpolynome. Sie erlaubt es, von der Symmetrie eines Potenzsummenpolynomes auf die des nächsten zu schließen.

Satz 2 Die Potenzsummenpolynome geraden Grades sind achsensymmetrisch zu $x=\frac{1}{2}$; Die Potenzsummenpolynome ungeraden Grades sind-von dem vom Grade 1 abgesehen - punktsymmetrisch zu $S\left(\frac{1}{2}, 0\right)$. 
Beweis. Wir beweisen die Behauptung durch vollständige Induktion. Sei zunächst $n=2$. Die Achsensymmetrie von $P_{2}$ ergibt sich durch eine einfache Rechnung. Sei nun $n \in \mathbb{N}$ mit $n \geq 2$ vorgegeben.

Sei zunächst $n$ ungerade. Nach Induktionsvoraussetzung ist $P_{n}$ ungerade bezüglich $\frac{1}{2}$. Daher folgt:

$$
P_{n+1}\left(\frac{1}{2}+x\right)-P_{n+1}\left(\frac{1}{2}-x\right)=n \int_{\frac{1}{2}-x}^{\frac{1}{2}+x} P_{n}(t) d t=0 .
$$

Daher ist $P_{n+1}$ gerade bezüglich $\frac{1}{2}$.

Sei nun $n$ gerade. Nach Induktionsvoraussetzung ist $P_{n}$ gerade bezüglich $\frac{1}{2}$. Daher folgt:

$$
\begin{aligned}
P_{n+1}\left(\frac{1}{2}+x\right)+P_{n+1}\left(\frac{1}{2}-x\right) & =n\left(\int_{0}^{\frac{1}{2}+x} P_{n}(t) d t+\int_{0}^{\frac{1}{2}-x} P_{n}(t) d t-\int_{0}^{1} P_{n}(t) d t\right) \\
& =n\left(\int_{\frac{1}{2}}^{\frac{1}{2}+x} P_{n}(t) d t+\int_{\frac{1}{2}}^{\frac{1}{2}-x} P_{n}(t) d t\right) \\
& =0 .
\end{aligned}
$$

Daher ist $P_{n+1}$ ungerade bezüglich $\frac{1}{2}$.

Wir bemerken, daß sich aus Satz 1 und Satz 2 in anschaulicher Weise eine wichtige Eigenschaft der Bernoullischen Zahlen ergibt:

Bemerkung Es gilt: $B_{3}=B_{5}=B_{7}=\cdots=0$.

\section{5 Über Nullstellen und Vorzeichen der Potenzsummenpolynome im Intervall $[0,1]$}

Einige Nullstellen der Potenzsummenpolynome sind offensichtlich: Das Polynom $P_{1}$ hat eine Nullstelle in 0 ; die Polynome $P_{2}, P_{3}, P_{4}, \ldots$ haben Nullstellen in 0 und in 1 ; die Polynome $P_{3}, P_{5}, P_{7}, \ldots$ haben eine Nullstelle in $\frac{1}{2}$. Wir bezeichnen diese Nullstellen als die trivialen Nullstellen der Potenzsummenpolynome.

Wir überlegen, ob die Potenzsummenpolynome im Intervall $[0,1]$ weitere Nullstellen besitzen. Dazu untersuchen wir diese Polynome auf Vorzeichen- und Wachstumseigenschaften in diesem Intervall. Wir bemerken, daß das Vorzeichen der Potenzsummenpolynome im Intervall $[0,1]$ noch aus einem weiteren Grunde interessant ist. Wir schließen aus diesem Vorzeichen auf die Mittelwerte der Potenzsummenpolynome in diesem Intervall. Diese Mittelwerte spielen aber in unserer Rekursionsformel eine wichtige Rolle.

Lemma 1 Die Polynome $P_{3}, P_{7}, P_{11}, \ldots$ sind positiv auf $] 0, \frac{1}{2}[$ und negativ auf $] \frac{1}{2}, 1[$. Die Polynome $P_{5}, P_{9}, P_{13}, \ldots$ sind negativ auf $] 0, \frac{1}{2}[$ und positiv auf $] \frac{1}{2}, 1[$. 
Beweis. Wir beweisen die Behauptung durch vollständige Induktion. Die Vorzeichenaussagen für $P_{3}$ weist man durch eine einfache Rechnung nach.

Sei $n \in \mathbb{N}$ ungerade mit $n \geq 3$. Aus der Rekursionsformel und weil $P_{n}$ ungerade bezüglich $\frac{1}{2}$ ist, ergibt sich:

$$
P_{n+2}^{\prime \prime}(x)=(n+1) n P_{n}(x)
$$

Sei o. B. d. A. $P_{n}$ positiv auf $] 0, \frac{1}{2}\left[\right.$. Dann ist $P_{n+2}$ auf $\left[0, \frac{1}{2}\right]$ konvex nach oben. Da außerdem $P_{n+2}$ in 0 und in $\frac{1}{2}$ Nullstellen besitzt, muß $P_{n+2}$ auf ]0, $\frac{1}{2}$ [ negativ sein. Aus Symmetriegründen ist dann $P_{n+2}$ positiv auf $] \frac{1}{2}, 1[$.

Lemma 2 Die Polynome $P_{2}, P_{6}, P_{10}, \ldots$ sind streng monoton fallend auf $\left[0, \frac{1}{2}\right]$ und streng monoton wachsend auf $\left[\frac{1}{2}, 1\right]$. Die Polynome $P_{4}, P_{8}, P_{12}, \ldots$ sind streng monoton wachsend auf $\left[0, \frac{1}{2}\right]$ und streng monoton fallend auf $\left[\frac{1}{2}, 1\right]$.

Beweis. Die Behauptung für $P_{2}$ rechnet man einfach nach. Nach der Rekursionsformel und aus Symmetriegründen gilt für alle geraden $n \in \mathbb{N}$ mit $n \geq 4$ :

$$
P_{n}^{\prime}(x)=(n-1) P_{n-1}(x) .
$$

Hieraus und aus Lemma 1 folgen die Behauptungen mit Hilfe des Globalen Wachstumssatzes.

Weil die Polynome $P_{2}, P_{4}, P_{6}, \ldots$ in 0 und in 1 Nullstellen besitzen und wegen der Wachstumsaussagen aus Lemma 2 ergibt sich weiter:

Lemma 3 Die Polynome $P_{2}, P_{6}, P_{10}, \ldots$ sind negativ auf $] 0,1\left[\right.$. Die Polynome $P_{4}, P_{8}$, $P_{12}, \ldots$ sind positiv auf $] 0,1[$.

Mit Hilfe von Lemma 1 und Lemma 3 können wir nun die Frage nach den Nullstellen der Potenzsummenpolynome im Einheitsintervall beantworten:

Satz 3 Die Potenzsummenpolynome besitzen im Intervall $[0,1]$ nur die trivialen Nullstellen.

Wir bemerken, daß sich aus Lemma 3 und Satz 1 wieder in anschaulicher Weise eine Folgerung über die Bernoullischen Zahlen ergibt:

Bemerkung Die $B_{2}, B_{4}, B_{6}, \ldots$ besitzen alternierende Vorzeichen.

\section{Die Vielfachheiten der trivialen Nullstellen}

Mit den trivialen Nullstellen der Potenzsummenpolynome befaßt sich übrigens das Problem 11 aus den Mathematischen Semesterberichten (vgl. die Problemstellung in [10] und die Lösungen in $[2 ; 3 ; 4 ; 7 ; 8 ; 9])$. Wir bemerken, daß wir mit diesem Aufsatz auch eine weitere und ganz elementare Lösung dieses Problems geben.

Satz 4 Die Polynome $P_{3}, P_{5}, P_{7}, \ldots$ besitzen in $0, \frac{1}{2}$ und in 1 Nullstellen der Vielfachheit 1 . 
Beweis. Sei $n \in \mathbb{N}$ ungerade mit $n \geq 3$. Nach der Rekursionsformel und wegen $P_{n}(1)=0$ gilt:

$$
P_{n}^{\prime}(1)=-(n-1) \int_{0}^{1} P_{n-1}(x) d x .
$$

Nach Lemma 3 ist der Mittelwert von $P_{n-1}$ auf $[0,1]$ nicht gleich 0 und daher ist $P_{n}^{\prime}(1) \neq 0$. Daher ist 1 Nullstelle der Vielfachheit 1 von $P_{n}$. Aus Symmetriegründen ist auch 0 Nullstelle der Vielfachheit 1 von $P_{n}$.

Nach der Rekursionsformel gilt ferner:

$$
P_{n}^{\prime}\left(\frac{1}{2}\right)=(n-1)\left(P_{n-1}\left(\frac{1}{2}\right)-\int_{0}^{1} P_{n-1}(x) d x\right) .
$$

Nach Lemma 2 kann der Mittelwert von $P_{n-1}$ auf $[0,1]$ nicht gleich dem Extremalwert $P_{n-1}\left(\frac{1}{2}\right)$ sein. Daher ist $P_{n-1}^{\prime}\left(\frac{1}{2}\right) \neq 0$ und somit auch $\frac{1}{2}$ Nullstelle der Vielfachheit 1 von $P_{n}$.

Satz 5 Das Polynom $P_{2}$ besitzt in 0 und in 1 Nullstellen der Vielfachheit 1. Die Polynome $P_{4}, P_{6}, P_{8}, \ldots$ besitzen in 0 und in 1 Nullstellen der Vielfachheit 2.

Beweis. Die Behauptung für $P_{2}$ beweist man durch einfache Rechnungen. Aus der Rekursionsformel und aus Symmetriegründen ergibt sich für alle geraden $n \in \mathbb{N}$ mit $n \geq 4$ :

$$
P_{n}^{\prime}(x)=(n-1) P_{n-1}(x) .
$$

Hieraus und aus Satz 4 ergeben sich die behaupteten Vielfachheiten der Nullstellen 0 und 1 von $P_{n}$.

\section{Bemerkung zu den nicht-trivialen Nullstellen}

Im allgemeinen besitzen die Potenzsummenpolynome außerhalb des Einheitsintervalles weitere Nullstellen. Z. B. gilt:

$$
\begin{aligned}
& P_{5}(x)=\frac{1}{5} x\left(x-\frac{1}{2}\right)(x-1)\left(x-\frac{1}{2}+\sqrt{\frac{7}{12}}\right)\left(x-\frac{1}{2}-\sqrt{\frac{7}{12}}\right), \\
& P_{6}(x)=\frac{1}{6} x^{2}(x-1)^{2}\left(x-\frac{1}{2}+\frac{\sqrt{3}}{2}\right)\left(x-\frac{1}{2}-\frac{\sqrt{3}}{2}\right) .
\end{aligned}
$$

Den Funktionsterm von $P_{7}$ in Faktoren zu zerlegen, ist schon etwas aufwendiger. Wir berücksichtigen, daß $P_{7}$ ungerade ist bezüglich $\frac{1}{2}$ und verwenden ein Computer-AlgebraSystem. Wir erhalten:

$$
P_{7}\left(\frac{1}{2}+x\right)=\frac{1}{7} x\left(x-\frac{1}{2}\right)\left(x+\frac{1}{2}\right)\left(\left(x^{2}-\frac{3}{4}\right)^{2}+\frac{1}{12}\right) .
$$

Hieraus folgt sofort, daß $P_{7}$ außer den trivialen Nullstellen keine weiteren reellen Nullstellen besitzt. Mit einer einfachen Überlegung können wir zeigen, daß dies auch für viele weitere Potenzsummenpolynome gilt: 
Satz 6 Die Polynome $P_{4}, P_{8}, P_{12}, \ldots$ besitzen nur die trivialen reellen Nullstellen.

Beweis. Aus der Definition der Potenzsummenpolynome folgt für alle $n \in \mathbb{N}$ mit $n \geq 2$ und für alle $x \in \mathbb{N}$ :

$$
P_{n}(x+1)=P_{n}(x)+x^{n-1} .
$$

Nach dem Identitätssatz für Polynome ist diese Beziehung sogar für alle $x \in \mathbb{R}$ erfüllt. Sei nun $n$ eine durch 4 teilbare natürliche Zahl. Dann besitzt $P_{n}$ nach Lemma 3 auf ]0, $[$ [ nur positive Werte. Hieraus und aus der obigen Beziehung schließt man weiter, daß $P_{n}$ auch rechts von 1 nur positive Werte hat. Aus Symmetriegründen besitzt $P_{n}$ dann auch links von 0 nur positive Werte.

\section{Literatur}

[1] J. Bernoulli: Ars conjectandi, Basel 1713.

[2] H. Braß: Lösung von Problem 11. Math. Semesterber. 41 (1994).

[3] G. Götsch: Lösung von Problem 11. Math. Semesterber. 31 (1984).

[4] W. Herget: Weitere Lösungen von Problem 11. Math. Semesterber. 31 (1984).

[5] H. Heuser: Lehrbuch der Analysis, Teil 1. Stuttgart: Teubner 1980.

[6] N. Kimura und H. Siebert: Über die rationalen Nullstellen der von den Potenzsummen der natürlichen Zahlen definierten Polynome. Proc. Japan Acad. Sci. 56 (1980).

[7] H. Länger: Teillösung zu Problem 11. Math. Semesterber. 41 (1994).

[8] P. Moore: Lösung von Problem 11. Math. Semesterber. 41 (1994).

[9] H. J. Seiffert: Lösung von Problem 11. Math. Semesterber. 41 (1994).

[10] U. Warnecke: Problem 11. Math. Semesterber. 30 (1983).

Dietmar Treiber

Libellenpfad 5

D-40764 Langenfeld 